\title{
Towards broadband electroacoustic resonators through optimized feedback control strategies
}

\author{
R. Boulandet ${ }^{\mathrm{a}}$, H. Lissek ${ }^{\mathrm{b}, *}$ \\ ${ }^{a}$ Groupe d'Acoustique de l'Université de Sherbrooke, 2500 Boul. de l'Université, J1K 2R1, \\ Québec, Canada \\ ${ }^{b}$ Laboratoire d'Electromagnétisme et d'Acoustique, Ecole Polytechnique Fédérale de \\ Lausanne, Station 11, CH-1015, Lausanne, Switzerland
}

\section{Abstract}

This paper presents a methodology for the design of broadband electroacoustic resonators for low-frequency room equalization. An electroacoustic resonator denotes a loudspeaker used as a membrane resonator, the acoustic impedance of which can be modified through proportional feedback control, to match a target impedance. However, such impedance matching only occurs over a limited bandwidth around resonance, which can limit its use for the lowfrequency equalization of rooms, requiring an effective control at least up to the Schroeder frequency. Previous experiments have shown that impedance matching can be achieved over a range of a few octaves using a simple proportional control law. But there is still a limit to the feedback gain, beyond which the feedback-controlled loudspeaker becomes non-dissipative. This paper evaluates the benefits of using PID control and phase compensation techniques to improve the overall performance of the electroacoustic resonator. More specifically, it is shown that some adverse effects due to high-order

\footnotetext{
* Corresponding author

Email address: herve.lissek@epfl.ch (H. Lissek)
} 
dynamics in the moving-coil transducer can be mitigated. The corresponding control settings are also identified with equivalent electroacoustic resonator parameters, allowing a straightforward design of the controller. Experimental results using PID control and phase compensation are finally compared in terms of sound absorption performances. As a conclusion the overall performances of electroacoustic resonators for damping the modal resonances inside a duct are presented, along with general discussions on practical implementation and the extension to actual room modes damping.

Keywords: Feedback-controlled loudspeaker, Electroacoustic resonator, Active sound absorption, Acoustic impedance control, Room modal equalization, PID control, Phase compensation.

1. Introduction

An increasing number of scientific publications report the development of 3 passive, semi-active and active techniques employing electroacoustic transduc4 ers as noise control "materials". Indeed, the moving parts of a loudspeaker 5 can readily be used as a membrane absorber, the acoustic absorption coefficient being maximal at resonance [1. Driven outside the linear range, the mechanical resonator can even be used as a nonlinear sound absorber, presenting the possibility to adjust the absorption according to the excitation [2]. Alternatively, the connection of an electrical resistance to the electrical terminals of a loudspeaker allows the absorption coefficient at resonance to be modified, ranging from the value achieved in open circuit, up to 1 [3, 4]. The frequency range of absorption can then be optimized through the selection of the electro-mechanical components of the system [5]. Similar techniques also 
allow sound insulation to be achieved, by connecting a negative capacitance to a clamped piezoelectric membrane, increasing its equivalent stiffness [6], or by loading an electrodynamic loudspeaker with a negative electric resistance, increasing the equivalent mechanical resistance of the resonator [7]. A loudspeaker employed as noise control material will be denoted an electroacoustic resonator in the following.

Electroacoustic resonators can be envisaged as a solution for room modes equalization [8], since conventional loudspeakers generally resonate in the very low-frequency range. Room modes equalization refers to the damping of low-frequency acoustic resonances inside a room, smoothing its response to an acoustic excitation over frequencies below the Schroeder frequency (eg. roughly within the frequency decade $[20-200 \mathrm{~Hz}]$ [9]). However, conventional passive materials have poor absorbing capabilities in this frequency range. Wedge-type absorbers used in anechoic chambers should be sufficiently deep to present enough absorption at low-frequencies, while bass-traps (membrane resonators) operate within a limited frequency range [1]. Thus, electroacoustic resonators can be seen as an adjustable alternative to bass-traps, but the bandwidth of such passive electroacoustic resonators is generally limited to a few tens of Hertz with conventional loudspeakers, which limits the applicability to room modes equalization.

Alternatively, active control methods have been developed for room modes equalization. Elliott et al. [10, 11] have obtained a significant reduction of the acoustic potential energy in a closed space, with a low modal density, by using secondary sources (loudspeakers). The latter generate anti-noise (with the same amplitude but opposite phase) to cancel out the noise from primary 
2 guided sound waves and stationary tones [12], but the treatment of complex 3 sound fields (broadband, non stationary sources) in large rooms is hardly 4 achievable with current ANC technologies. The number of secondary sources 5 to be deployed becomes prohibitive and the distributed control algorithms 6 may be too complicated to implement [13], reducing its interest for equalizing 7 rooms for music diffusion.

8 The direct impedance control technique [14] can be considered as an

sources at some sensors positions. Good performance can be achieved for active counterpart to passive electroacoustic resonators. This technique aims at imposing a specified acoustic impedance over a loudspeaker diaphragm, through a closed-loop proportional feedback control employing a combination of sound pressure and velocity sensing. The ratio of the feedback gains determines the value of the target acoustic impedance at the diaphragm, which is set so as to match the characteristic impedance of the medium. In practice the achieved absorption performance can be significantly extended along a broader frequency bandwidth compared to passive electroacoustic resonators [4, 15]. However, such active strategies are generally limited by a side effect of the Bode's sensitivity integral theorem [16]. This side effect is essentially characterized by a frequency-dependent overshoot in the sound pressure reflected by the loudspeaker diaphragm, thus limiting such a technique. To ensure broad sound absorption with such technique, thus allowing its use for room mode equalization, this overshoot effect needs to be compensated for with advanced control strategies.

The present paper investigates such compensation strategies for a direct impedance control system achieving broadband electroacoustic resonators out 
1 of conventional loudspeakers, for application to room modes equalization. We 2 will consider here only 1 dimensional guided waves, for the sake of simplicity. 3 By minimizing an error signal derived from the sensed quantities, a controlled 4 acoustic impedance is achieved at the loudspeaker diaphragm. To obtain 5 perfect sound absorption over a desired frequency bandwidth, the loudspeaker 6 impedance should be targeting the constant characteristic acoustic resistance $7 \rho c, \rho$ and $c$ being the mass density and sound velocity of the medium. But 8 here, a frequency-dependent reactance will also be accounted for with a view 9 to improving the control stability and the absorption performances. The main 10 objective of the paper is to compare different control techniques outperforming the simple proportional control, especially in terms of bandwidth extension. This paper addresses PID architectures, as well as phase compensation strategies. Furthermore, this paper compares the different control strategies through the introduction of performance indicators. At last, an original methodology for the design of broadband electroacoustic resonators is provided, that can be extended to $3 \mathrm{D}$ contexts. The remainder of the paper is organized as follows. First, a circuit equivalent model of the electroacoustic resonator is presented. A baseline control scheme for achieving active sound absorption is then discussed, using a frequency response approach. The control strategy is then extended to PID control and phase compensation, which are thoroughly described and compared. The emphasis is put on increasing the sound absorption capability in terms of bandwidth and stability. Experimental results follow, illustrating the performance of a feedback-controlled loudspeaker to dampen the low-frequency modal resonances in a duct. Concluding remarks are presented along with a general discussion on the practical implementation 
1

and extension to 3D enclosed sound fields.

\section{Electroacoustic resonator: concept and formulation}

\subsection{Description and model of the electrodynamic loudspeaker}

An electrodynamic loudspeaker can be considered as a single-degree-offreedom mechanical oscillator that is driven by a voice coil within a permanent and almost constant magnetic field. Referring to Fig. 1, the mechanical part can be represented by a mass-spring-damper system in the low-frequency range. It is assumed that all forces acting on the transducer are small enough to consider the loudspeaker as a linear, time-invariant dynamic system, otherwise, some of the quantities representing the transducer dynamics may become time-varying and nonlinear functions of the excitation [17, 18]. The electrical parts of the system are modeled through linear circuits, including the electromechanical coupling. As shown in Fig. 1, the mechanical and electrical part are connected together by means of an electrodynamic transducer whose constitutive equations can be obtained from Faraday's law and Lorentz's law [19.

Let's denote $S$ the effective piston area, and $B l$ the force factor of the moving-coil transducer. The electrical quantities $L_{e}$ and $R_{e}$ represent the self-inductance and the dc resistance of the coil. The quantities $M_{m s}, R_{m s}$ and $C_{m s}$ represent the moving mass, the mechanical resistance and the mechanical compliance accounting for the elastic surround suspension and the spider. The preceding quantities are commonly denoted the Thiele-Small parameters of the loudspeaker [20]. Note that these mechanical quantities also account for fluid-structure coupling at the diaphragm. If the loudspeaker is loaded by a 


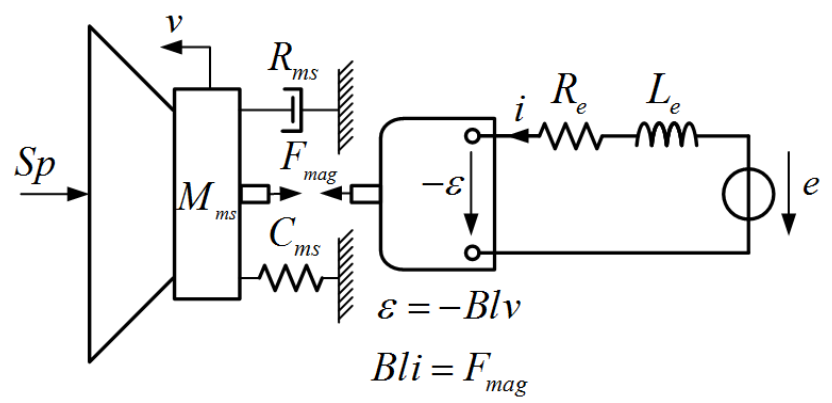

Figure 1: Schematic representation of an electrodynamic loudspeaker with terms of electromechanical coupling included: $F_{m a g}=B l i$ is the force of electric origin resulting from the magnetic field acting on a moving free charge (current), and $\varepsilon=-B l v$ is the back electromotive force (voltage) induced by the motion of the voice coil within the magnetic field.

5 compliance $C_{m c}=C_{m s} C_{m b} /\left(C_{m s}+C_{m b}\right)[21]$. 6 The equation of motion follows from Newton's second law and can be 7 written as

$$
S p(t)+B l i(t)-R_{m s} v(t)-\frac{1}{C_{m c}} \int v(t) \mathrm{d} t=M_{m s} \dot{v}(t)
$$

8

rear volume $V_{b}$, the reaction of the fluid that acts on the rear face is commonly accounted for in an equivalent mechanical compliance $C_{m b}=V_{b} /\left(\rho c^{2} S^{2}\right)$. In the following, we will consider the system composed of the loudspeaker and a closed-box of volume $V_{b}$, thus introducing the equivalent mechanical where $p$ is the input sound pressure acting on the diaphragm, $v$ is the diaphragm velocity and $i$ is the electrical current flowing through the voice coil.

The governing equation of the electrical side is based on Kirchhoff's laws 
1 and can be written as

$$
e(t)=R_{e} i(t)+L_{e} \dot{i}(t)+B l v(t)
$$

2 where $e$ is the input voltage between the electric terminals. Note that the 3 model given in Eq. (2) can be further detailed by taking into account eddy 4 currents which are created when a conductor (here the voice coil) experiences 5 changes in the magnetic field, in accordance with Faraday's law of induction. 6 These phenomena yield substantial changes in the magnitude and the phase 7 relationship between voltage and current in $L_{e}$ [17, 18]. For the sake of 8 simplicity, these effects are not taken into account in what follows.

9 written as

$$
\begin{aligned}
S P(s) & =\left(s M_{m s}+R_{m s}+\frac{1}{s C_{m c}}\right) V(s)-B l I(s) \\
E(s) & =\left(R_{e}+s L_{e}\right) I(s)+B l V(s)
\end{aligned}
$$

13 where $s=j \omega$ is the Laplace variable, and where all capital letters represent the

Taking the Laplace transform of Eqs. (1) and (2) yields the coupled set of characteristic equations of the electrodynamic loudspeaker, which can be Laplace transform of the corresponding time-domain quantity in minuscules. Note that the outward current is shown as positive, and the inward velocity is considered as positive.

\subsection{Coupling to an auxiliary voltage source}

Eq. (3) provides the general model of an electrodynamic loudspeaker. Driven by an auxiliary voltage source $E_{g}$, the voltage $E$ at the loudspeaker terminals is

$$
E(s)=E_{g}(s)-Z_{L}(s) I(s)
$$


Table 1: Thiele-Small parameters of the Visaton ${ }^{\circledR}$ AL-170 loudspeaker.

\begin{tabular}{lccc}
\hline Parameter & Notation & Value & Unit \\
\hline Nominal Impedance & $Z$ & 8 & $\Omega$ \\
Resonance frequency & $f_{s}$ & 38 & $\mathrm{~Hz}$ \\
Moving mass & $M_{m s}$ & 13 & $\mathrm{~g}$ \\
Mechanical resistance & $R_{m s}$ & 0.8 & $\mathrm{~N} \mathrm{~s} \mathrm{~m}^{-1}$ \\
Mechanical compliance & $C_{m s}$ & 1.35 & $\mathrm{~mm} \mathrm{~N}^{-1}$ \\
dc resistance of the coil & $R_{e}$ & 5.6 & $\Omega$ \\
Self-inductance of the coil & $L_{e}$ & 0.9 & $\mathrm{mH}$ \\
Force factor & $B l$ & 6.9 & $\mathrm{~T} \mathrm{~m}$ \\
Effective piston area & $S$ & 133 & $\mathrm{~cm}^{2}$ \\
\hline
\end{tabular}

1 where $Z_{L}$ is the source impedance.

2 Combining Eq. (3) and Eq. (4), the normal velocity $V$ of the diaphragm 3 can be derived as a function of pressure $P$ and voltage $E$

$$
V(s)=\frac{S\left(Z_{e}(s)+Z_{L}(s)\right)}{Z_{m}(s)\left(Z_{e}(s)+Z_{L}(s)\right)+(B l)^{2}} P(s)+\frac{B l}{Z_{m}(s)\left(Z_{e}(s)+Z_{L}(s)\right)+(B l)^{2}} E_{g}(s)
$$

4 where $Z_{m}(s)=s M_{m s}+R_{m s}+1 /\left(s C_{m c}\right)$ is the mechanical impedance and ${ }_{5} Z_{e}(s)=R_{e}+s L_{e}$ is the blocked electrical impedance of the loudspeaker. Eq. 6 (5) indicates that the diaphragm velocity in response to an incident pressure $7 \quad P(s)$ can be controlled by applying an adjustable voltage function $E(s)$ to 8 the voice coil. For the sake of simplicity, the source internal impedance $Z_{L}(s)$ 9 will be neglected in the following, yielding $E_{g}(s)=E(s)$ and

$$
V(s)=D(s) P(s)+G(s) E(s)
$$




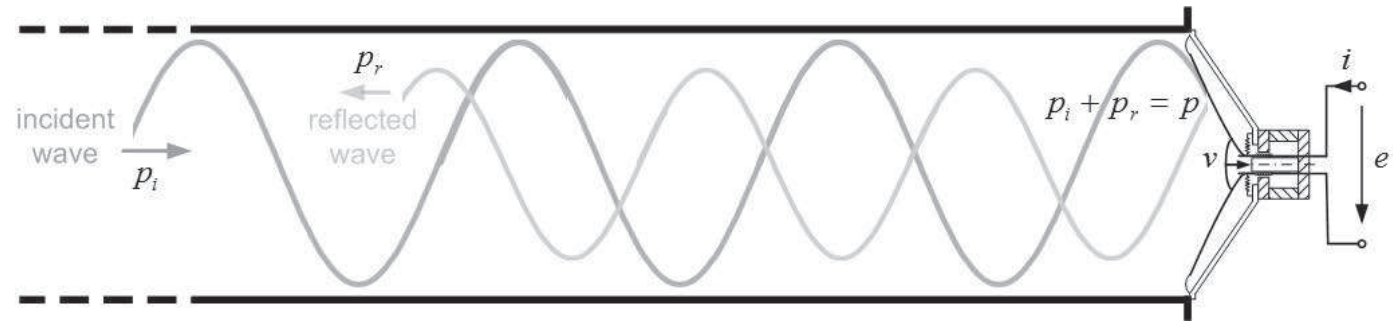

Figure 2: Semi-infinite wave guide that emulates an acoustical transmission line. The cone loudspeaker shown on the right-hand side is seen as an acoustic load for the incident plane waves. The total sound pressure $p$ acting on the diaphragm is the sum of incident $p_{i}$ and reflected $p_{r}$ sound pressures.

1 where $D(s)=S Z_{e}(s) /\left(Z_{m}(s) Z_{e}(s)+(B l)^{2}\right)$ and $G(s)=B l /\left(Z_{m}(s) Z_{e}(s)+\right.$ $\left.2(B l)^{2}\right)$.

\section{3. Baseline control strategy for acoustic impedance matching}

\section{3.1. Direct control of acoustic impedance}

5 Let us consider the loudspeaker of Fig. 1 used as a membrane absorber 6 and placed at the termination of a semi-infinite wave guide of adapted cross7 section, as shown in Fig. 2, A rigid-walled duct, the lateral dimensions of 8 which are small enough compared to the wavelength, can be considered as 9 a non-dissipative acoustical transmission line [22]. The loudspeaker, which plays the role of an acoustic load at the end of the transmission line, can be characterized by a complex-valued specific impedance $Z=\frac{p}{v}$, as defined in [23]. According to transmission line theory, the absorption condition consists in maximizing the acoustic energy transfer between the acoustic field in the duct and the loudspeaker diaphragm. To achieve such a condition at this termination, the specific impedance of the diaphragm must be set so as to 
1

perfectly match the transmission line characteristic impedance. This means that the ratio of the incident acoustic pressure and the output diaphragm velocity must be real and ideally equal to $\rho c$, and therefore the phase difference between these two variables should be zero. However, the reactances in the electroacoustic transducer dynamics induce a frequency-dependent phase shift between pressure and diaphragm velocity, that must be compensated through active means in order to increase the sound absorption capability, especially in terms of bandwidth. More specifically, both mechanical reactances (suspension compliance $C_{m s}$ and dynamic mass $M_{m s}$ ) in the loudspeaker impedance cause the output diaphragm velocity to be out-of-phase with the input sound pressure by $\pi / 2$ and $-\pi / 2$, respectively, away from the loudspeaker mechanical resonance. In addition, the self-inductance of the coil $L_{e}$ also induces an inflexion in the negative phase shift above the resonance frequency, when the loudspeaker is connected to an electric circuit [4].

From a control theory perspective, the matching condition can be reformulated through an error signal $\varepsilon$ to be minimized by a controller [14], that can be written as

$$
\varepsilon(t)=\frac{p(t)}{\rho c}-v(t)
$$

where $p / \rho c$ is the time-varying reference (set point) that is proportional to the driving acoustic pressure, and $v$ is the measured process variable, i.e. the output diaphragm velocity.

Figure 3 illustrates the block diagram of such feedback control strategy. In case of proportional control, i.e. $H(s)=K_{p}$ where $K_{p}$ if a feedback gain, the control signal can be expressed as a linear combination of the sound pressure 
1 and vibratory velocity sensed at the diaphragm [4], as

$$
e(t)=K_{p}\left(\frac{p(t)}{\rho c}-v(t)\right)
$$

2 The action of the controller thus consists in ensuring that the diaphragm 3 vibrates with the same value as the reference input $p / \rho c$, or, in other words, 4 in keeping the tracking error $\varepsilon$ as small as possible. Transposed to a frequency 5 response approach, this implies that the phase shift between the reference 6 input and output velocity should be zero in the bandwidth of interest. Then, 7 the smaller the phase shift, the broader the bandwidth of acoustic impedance 8 matching. The primary goal of the control system is therefore to adjust the 9 acoustic resistance of the diaphragm by increasing the mechanical damping, then to minimize the effects of the residual reactive parts so as to extend the bandwidth over which the impedance matching occurs.

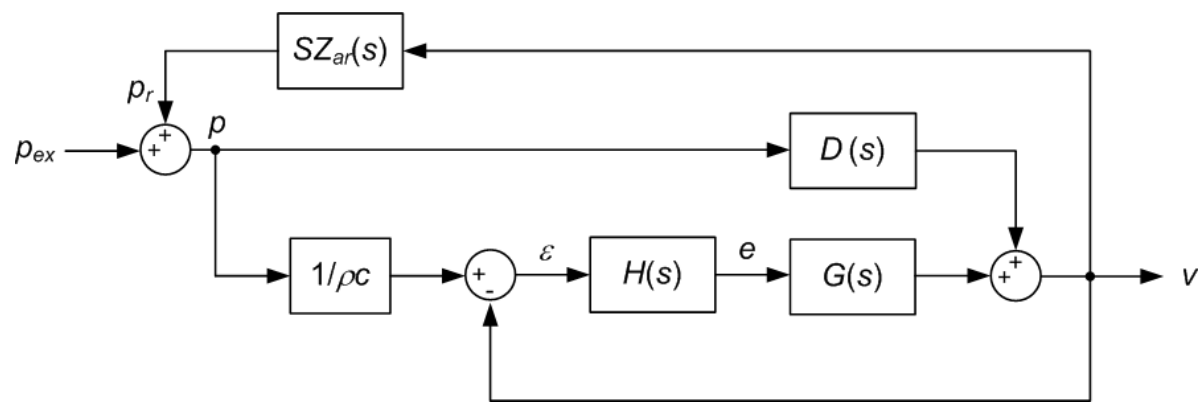

Figure 3: Block diagram of the electrodynamic loudspeaker system under feedback control.

12 Real world applications for such membrane absorbers, such as room 13 mode equalization, generally require frequency-dependent, complex-valued functions of the target impedance. This is not necessarily reflected in Eq. (8) where a real-valued setpoint, i.e. the characteristic impedance of air $\rho c$, is targeted. Since this study is investigating optimizing strategies to achieve 
sound absorption out of conventional loudspeakers (and not strictly targeting optimal acoustic performances in all situations), $\rho c$ will still be used as a target in the following, which will present the advantage of simplicity. The challenge of the following optimization is then to match the complex-valued impedance of the loudspeaker diaphragm to the real-valued characteristic impedance of air, over a broad frequency range.

\subsection{Acoustic absorption capability}

The closed-loop transfer function $V / P$ depicted in Fig. 3 can be derived from the characteristic equations Eq. (3) and the command voltage applied across the terminals of Eq. (4). Substituting the Laplace transform of Eq. (8) in Eq. (3) yields the closed form expression of the specific acoustic admittance, defined as

$Y_{P}(s)=\frac{V(s)}{P(s)}=\frac{S L_{e} s^{2}+\left(S R_{e}+B l K_{p} / \rho c\right) s}{M_{m s} L_{e} s^{3}+\left(M_{m s} R_{e}+R_{m s} L_{e}\right) s^{2}+\left(R_{m s} R_{e}+\frac{L_{e}}{C_{m c}}+(B l)^{2}+B l K_{p}\right) s+\frac{R_{e}}{C_{m c}}}$

This complex-valued, frequency-dependent quantity describes the dynamic response of the diaphragm to an external acoustic disturbance, characterizing the acoustic properties of the surface. Also, Eq. (9) clearly shows that the value of the feedback gain $K_{p}$ will affect the coefficients in the numerator and denominator, thereby modifying both the system gain and closed loop poles. The sound absorption coefficient $\alpha$ can then be derived as

$$
\alpha=1-\left|\frac{1-\rho c Y(s)}{1+\rho c Y(s)}\right|^{2}
$$

and defines the ratio of the acoustic power absorbed by the surface of materials (here the loudspeaker cone) with respect to normal incidence sound power 
1 24.

\section{3.3. Dynamics of a third-order system}

3 The self inductance of the coil is usually neglected in loudspeaker design 4 at low frequencies [21, 25]. Assuming the loudspeaker is driven by the input 5 voltage given by Eq. (8), the low-frequency approximation of the transfer

6 function $V / P$ is of second order. Therefore, the loudspeaker system takes the 7 form of a single-degree-of-freedom resonator [4, 25]

$$
Y_{P}(s)=K \frac{s}{s^{2}+2 \zeta \omega_{0} s+\omega_{0}^{2}}
$$

8 which can be characterized by three parameters

$$
K=\frac{S R_{e}+B l K_{p} / \rho c}{M_{m s}} \quad \omega_{0}=\frac{1}{\sqrt{M_{m s} C_{m c}}} \quad \zeta=\frac{R_{m s} R_{e}+(B l)^{2}+B l K_{p}}{2 M_{m s} R_{e} \omega_{0}}
$$

9 where $K$ is the system gain (in $\mathrm{m} \mathrm{s}^{-1} \mathrm{~Pa}^{-1}$ ), $\omega_{0}$ is the natural resonance

$$
b_{3}=M_{m s} L_{e} \quad b_{2}=M_{m s} R_{e}+R_{m s} L_{e} \quad b_{1}=R_{m s} R_{e}+\frac{L_{e}}{C_{m c}}+(B l)^{2}+B l K_{p} \quad b_{0}=\frac{R_{e}}{C_{m c}}
$$

15 are positive real coefficients. As detailed in the Appendix (see also [26, 27]

16 for further details), Eq. (13) can be factorized as

$$
Q(s)=\left(s-p_{1}\right)\left(s-p_{2}\right)\left(s-p_{3}\right)
$$


1 and after some further manipulations, the roots of Eq. (13) are given by

$$
Q(s)=(s+\sigma)\left(s+\zeta \omega_{t}+j \omega_{t} \sqrt{1-\zeta^{2}}\right)\left(s+\zeta \omega_{t}-j \omega_{t} \sqrt{1-\zeta^{2}}\right)
$$

2 where

$$
\sigma=-p_{1} \quad 2 \zeta \omega_{t}=-p_{2}-p_{3} \quad \omega_{t}^{2}=p_{2} p_{3}
$$

3

Then, Eq. (9) can be written as

$$
Y_{P}(s)=K \frac{s(s+\nu)}{(s+\sigma)\left(s^{2}+2 \zeta \omega_{t} s+\omega_{t}^{2}\right)}
$$

${ }_{4}$ where $K=S / M_{m s}$, and $\nu=R_{e} / L_{e}+B l K_{p} /\left(\rho c S L_{e}\right)$. As shown in Eq. 18), 5 the loudspeaker system has one real pole and two complex conjugate poles.

6 The damping ratio $\zeta$ in Eq. (18) can then be increased through the feedback 7 control gain $K_{p}$, in order to achieve sound absorption over a broader frequency 8 bandwidth. Then, the action of the proportional feedback control can be 9 interpreted as adding an equivalent mechanical resistance to the system.

\subsection{Stability of the feedback-controlled system}

In accordance with the Routh's criterion [28, the necessary condition for stability implies that all poles in Eq. (18) must have negative-real values. It means that the coefficients $b_{3}, b_{2}, b_{1}, b_{0}$ in Eq. (13) must be positive and verifying $b_{2} b_{1}>b_{3} b_{0}$. With the proportional control of Eq. (8), the closed-loop system is stable when

$$
K_{p}>\frac{1}{B l}\left[\frac{R_{e} M_{m s} L_{e}}{C_{m c}\left(M_{m s} R_{e}+R_{m s} L_{e}\right)}-R_{m s} R_{e}-\frac{L_{e}}{C_{m c}}\right]-B l
$$

i.e., as long as the feedback gain is positive. This is consistent with the control objective mentioned in the preceding section, aiming at providing additional damping to the system. 
1 3.5. Adverse effect due to high-order dynamics in the loudspeaker system.

2 An interesting, yet problematic, phenomenon always occurs when applying 3 proportional control with positive gain. While increasing the feedback gain,

4 the electroacoustic resonator may no longer remain a purely dissipative system.

5 Around a certain frequency, the active electroacoustic resonator can reflect 6 more energy than that provided by the sound field. The power supplied from 7 the control system is then greater than the power dissipated, thus resulting 8 in an overshoot in the system response [16]. This adverse effect, which 9 increases as feedback gains increase, is mainly due to high-order coefficients in the loudspeaker admittance, especially due to the voice-coil inductance occurring in the high-frequency range. To ensure that the system strictly dissipates energy over the full range, the closed-loop transfer function $Y_{P}$ in Eq. (9) must be such that $\operatorname{Re}\left[Y_{P}(j \omega)\right] \geqslant 0$ for all $\left.\omega \in\right]-\infty,+\infty[$ [29]. not necessarily in the whole frequency range of interest, as illustrated in Fig.

16 4. The "crossover" frequency, corresponding to the frequency at which the phase of the normalized admittance intersects the bound $-\pi / 2$ in Fig. 4(a), can be deduced from Eqs. 15, 18). As can also be seen on Fig. 4(b), this accident on the admittance phase yields negative absorption coefficient above the "crossover" frequency. The next section focuses on advanced control techniques to mitigate this problem. 


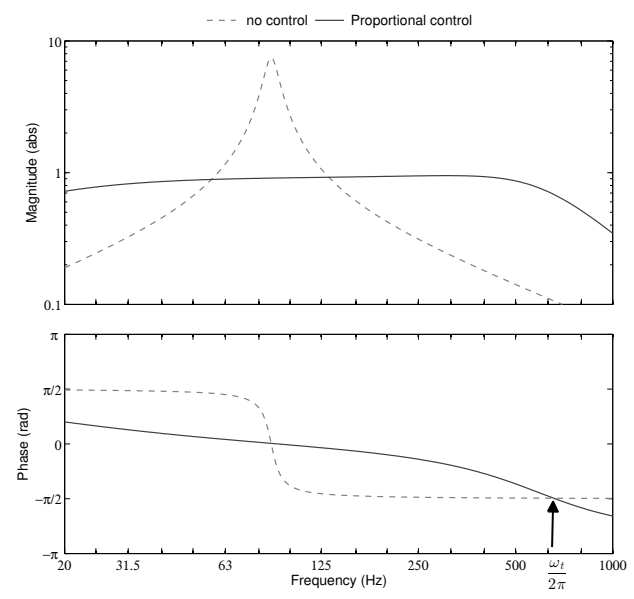

(a)

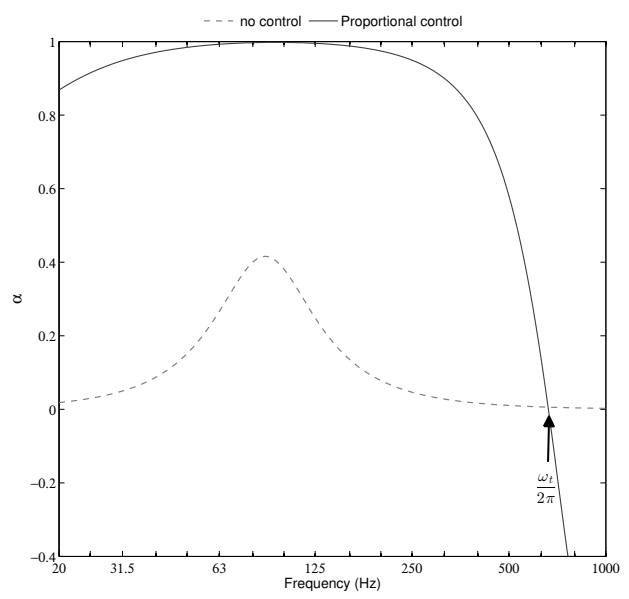

(b)

Figure 4: Bode plot (a) of the specific acoustic admittance $Y_{P}$ and acoustic absorption coefficient (b) of the electroacoustic resonator computed without control (open-circuit) and under proportional control ; $\omega_{t}$ (see Eq. (18)) is the crossover frequency from which the feedback-controlled loudspeaker starts to be non-dissipative.

4. Methodology for designing advanced control system for electroacoustic resonators

\section{4.1. PID controller}

$4 \quad$ Proportional-Integral-Derivative (PID) controllers are widely used in in-

5 dustrial control applications to obtain a desired response [28, 30]. Assuming

6 an error signal $\varepsilon$, the usual form of a PID controller output $e$ is given by

$$
e(t)=K_{p}\left(\varepsilon(t)+\frac{1}{T_{i}} \int_{0}^{t} \varepsilon(\tau) \mathrm{d} \tau+T_{d} \frac{\mathrm{d}}{\mathrm{d} t} \varepsilon(t)\right)
$$

7 where $K_{p}$ is the proportional gain, $T_{i}$ is the integral time constant, and $T_{d}$ is 8 the derivative time constant. As shown in Eq. 20), the PID control algorithm 9 basically involves three separate constant parameters which are respectively 
1 proportional to the error $\varepsilon$ at the instant $t$, to the integral of the error $\varepsilon$ up to 2 instant $t$, and to the derivative of the error $\varepsilon$ at instant $t$. The corresponding 3 transfer function of the PID controller can then be written as

$$
C_{P I D}(s)=K_{p}\left(1+\frac{1}{T_{i} s}+T_{d} s\right)
$$

4 The block diagram of the loudspeaker system under PID control can be 5 obtained after substituting $C_{P I D}(s)$ for $H(s)$ in Fig. 3 . It is worth mentioning 6 that Eq. (21) can not be implemented in practice since the transfer function of 7 the controller is improper. Moreover, the high-frequency gain of the derivative 8 term amplifies the measurement noise in the manipulated variable $e$. These 9 problems can be solved by applying a low-pass filtering to the derivative 10 action. As a result, the transfer function of Eq. 21) is modified as

$$
C_{P I D}(s)=K_{p}\left(1+\frac{1}{T_{i} s}+\frac{T_{d} s}{1+T_{d} s / N}\right)
$$

11 where $N$ is the filter time constant that should be selected so that the high12 frequency noise is suitably filtered without influencing the dominant dynamics 13 of the PID controller significantly [30]. Substituting the PID controller 14 function given in Eq. (22) for $E(s)$ in Eq. (6) gives the closed form expression 15 of the specific acoustic admittance as

$$
Y_{P I D}(s)=\frac{V(s)}{P(s)}=\frac{a_{2} s^{2}+a_{1} s+a_{0}}{b_{3} s^{3}+b_{2} s^{2}+b_{1} s+b_{0}}
$$

16 where

$$
\begin{array}{ll}
a_{2}=S L_{e}+(B l / \rho c) T_{d} & b_{3}=M_{m s} L_{e} \\
a_{1}=S R_{e}+(B l / \rho c) K_{p} & b_{2}=M_{m s} R_{e}+R_{m s} L_{e}+B l T_{d} \\
a_{0}=(B l / \rho c) \frac{1}{T_{i}} & b_{1}=R_{m s} R_{e}+\frac{L_{e}}{C_{m c}}+(B l)^{2}+B l K_{p} \\
b_{0} & =\frac{R_{e}}{C_{m c}}+\frac{B l}{T_{i}}
\end{array}
$$


1 Apart from the coefficient $b_{3}$, the PID parameters $\left(K_{p}, T_{i}, T_{d}\right)$ are formally 2 present in each of the other coefficients, thereby allowing to further modify 3 the loudspeaker dynamics. In addition to the action of the proportional term 4 in $b_{1}$ discussed in section 3.3 , the action of the integral term $T_{i}$ consists in 5 modifying the total active compliance (in $b_{0}$ ), and the action of the derivative 6 term modifies the total active mass (in $b_{2}$ ). The global action of the PID 7 controller can then be assimilated to an active mass-compliance-damping 8 resonator, the control gains allowing the modification of each mechanical 9 parameter individually.

10 Figure 5 (a) illustrates the Bode plot of the PID controller transfer function

$C_{P I D}$, the parameters of which can be found in Tab. 2. Note that the integral and derivative constants have been selected in such a way that the resonance frequency is kept the same than with no control. Unlike proportional control, PID control can then implement a frequency-dependent function as the target impedance. As shown in Fig. 5(a), the controller gain can be slightly increased below and above the resonant frequency of the loudspeaker. In addition, the PID controller should be designed to further reduce the phase shift between the driving pressure and diaphragm velocity in the frequency range of interest. Therefore, it is expected that the PID controller can improve the bandwidth where the impedance matching is effective, thereby improving the diaphragm absorption capabilities. Depending on the specifications it is not necessary to involve all the three control terms. A simple PI or PD control law may be sufficient to achieve broadband impedance matching. 


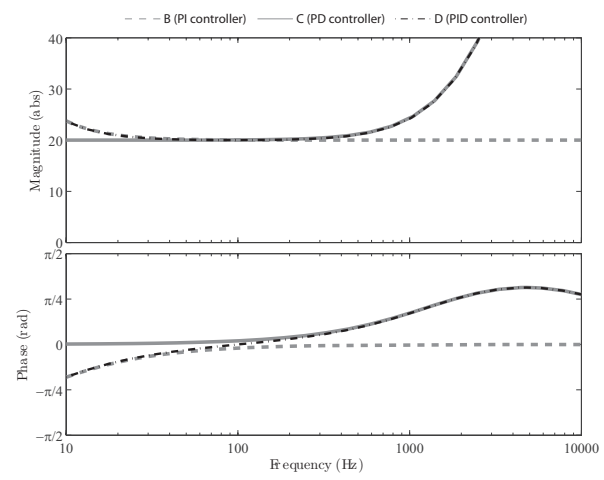

(a)

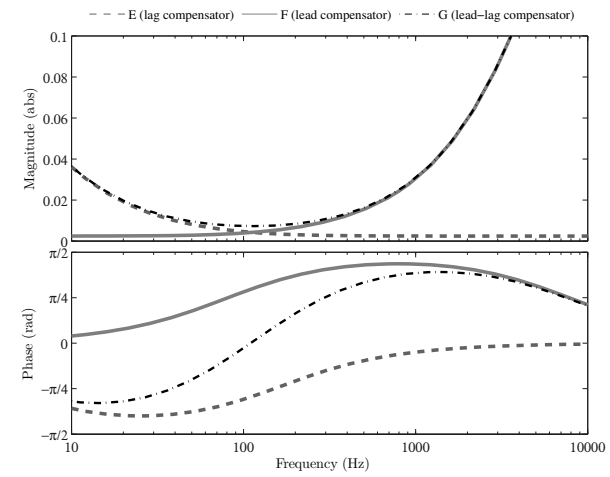

(b)

Figure 5: Bode plot of magnitude and phase for the PID transfer function (left) and for the transfer functions of lead/lag compensators (right).

1

2

9 function, is given by

$$
\begin{aligned}
C_{\text {lead/lag }}(s) & =K_{c}\left(\frac{1}{\gamma} \frac{s+z_{d}}{s+p_{d}}\right) \cdot\left(\frac{1}{\beta} \frac{s+z_{g}}{s+p_{g}}\right) \\
& =K_{c} \frac{\tau_{d} s+1}{\gamma \tau_{d} s+1} \cdot \frac{\tau_{g} s+1}{\beta \tau_{g} s+1} \quad \text { with } \quad 0<\gamma<1 \quad \text { and } \beta \geq 1
\end{aligned}
$$

10 This control architecture consists of a gain $K_{c}$, two poles $\left(p_{d}=1 / \gamma \tau_{d}\right.$ 11 and $\left.p_{g}=1 / \beta \tau_{g}\right)$ and two zeros $\left(z_{d}=1 / \tau_{d}\right.$ and $\left.z_{g}=1 / \tau_{g}\right)$. It is the series 
combination of a lag compensator (labelled with the subscript $g$ ) and a lead compensator (labelled with the subscript $d$ ). Figure 5 (b) illustrates the typical frequency response $C_{\text {lead/lag }}$ of a lag compensator (case E), a lead compensator (case F) and a lead/lag compensator (case G), the parameters of which can be found in Tab. 2,

One of the most important characteristics of the lead compensator is the positive phase shift in the intermediate frequencies, as illustrated in Fig. 5 (b). However, it also increases the gain of the system at high frequencies, meaning that high-frequency noise can be amplified. The frequency $f_{d}$ (in $\mathrm{Hz}$ ) at which the maximum phase-lead occurs, and the maximum phase-lead value $\phi_{d}$ in degrees are given by

$$
f_{d}=\frac{1}{2 \pi} \frac{1}{\tau_{d} \sqrt{\gamma}} \quad \text { and } \quad \phi_{d}=\frac{180}{\pi} \arcsin \left(\frac{1-\gamma}{1+\gamma}\right)
$$

Likewise, lag compensators are characterized by a constant attenuation in magnitude and a zero phase shift at high frequencies. As illustrated in Fig. 5 (b), a large negative phase shift is also expected at intermediate frequencies. The frequency $f_{g}$ (in $\mathrm{Hz}$ ) where the maximum phase-lag occurs, and the maximum phase-lag value $\phi_{g}$ in degrees are given by

$$
f_{g}=\frac{1}{2 \pi} \frac{1}{\tau_{g} \sqrt{\beta}} \text { and } \quad \phi_{g}=\frac{180}{\pi} \arcsin \left(\frac{1-\beta}{1+\beta}\right)
$$

Figure 6 shows the block diagram of the feedback-controlled loudspeaker including a phase lead/lag compensator. To limit the overshoot in the output velocity resulting from the feedback control, it is necessary to ensure that the response of the electroacoustic resonator remains as close as possible to its dynamics without control out of the bounds of the frequency range of interest. In the context of impedance matching this implies, among other things, that 


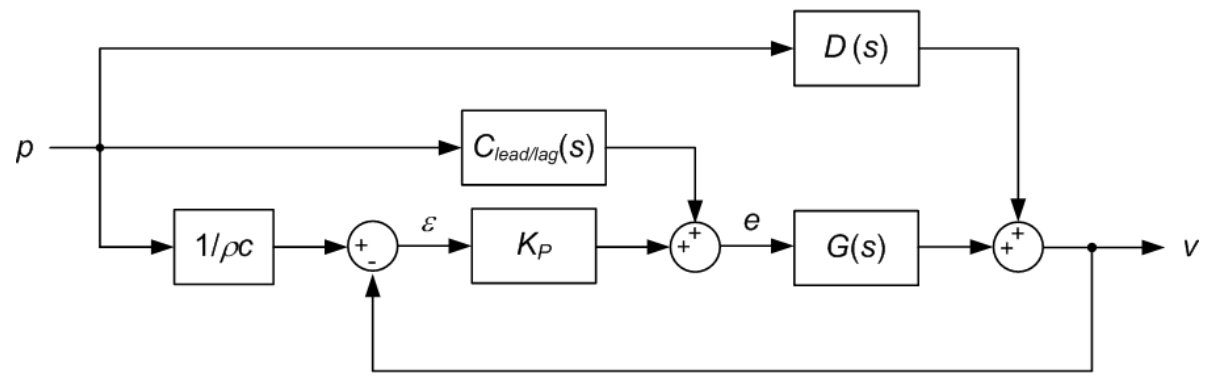

Figure 6: Block diagram of the feedback-controlled loudspeaker including a phase compensation.

1 the phase between the driving pressure and output diaphragm velocity must 2 remain between $-\pi / 2$ and $\pi / 2$. To this end, a phase lead compensation can 3 be highly relevant when the phase shift exceeds $-\pi / 2$. In addition, a phase 4 lag compensation can decrease the phase shift below the natural resonance of 5 the loudspeaker, thereby further increasing the bandwidth. These two aspects 6 will be discussed in the next section.

7

8 The closed-form expression of the specific acoustic admittance as given in 9 Fig. 6, can be derived as

$$
Y_{\text {lead } / \text { lag }}(s)=\frac{V(s)}{P(s)}=\frac{a_{4} s^{4}+a_{3} s^{3}+a_{2} s^{2}+a_{1} s+a_{0}}{b_{5} s^{5}+b_{4} s^{4}+b_{3} s^{3}+b_{2} s^{2}+b_{1} s+b_{0}}
$$


1 where

$$
\begin{aligned}
& a_{4}=S L_{e}\left(\gamma \beta \tau_{d} \tau_{g}\right) \\
& a_{3}=S L_{e}\left(\alpha \tau_{d}+\beta \tau_{g}\right)+\left(S R_{e}+(B l / \rho c) K_{p}\right)\left(\gamma \beta \tau_{d} \tau_{g}\right)+(B l / \rho c) K_{c} \tau_{d} \tau_{g} \\
& a_{2}=S L_{e}+\left(S R_{e}+(B l / \rho c) K_{p}\right)\left(\gamma \tau_{d}+\beta \tau_{g}\right)+(B l / \rho c) K_{c}\left(\tau_{d}+\tau_{g}\right) \\
& a_{1}=S R_{e}+(B l / \rho c)\left(K_{p}+K_{c}\right) \\
& b_{5}=L_{e} M_{m s}\left(\gamma \beta \tau_{d} \tau_{g}\right) \\
& b_{4}=L_{e} M_{m s}\left(\gamma \tau_{d}+\beta \tau_{g}\right)+\left(L_{e} R_{m s}+R_{e} M_{m s}\right)\left(\gamma \beta \tau_{d} \tau_{g}\right) \\
& b_{3}=L_{e} M_{m s}+\left(L_{e} R_{m s}+R_{e} M_{m s}\right)\left(\gamma \tau_{d}+\beta \tau_{g}\right)+\left(R_{e} R_{m s}+L_{e} / C_{m c}+(B l)^{2}+B l K_{p}\right)\left(\gamma \beta \tau_{d} \tau_{g}\right) \\
& b_{2}=L_{e} R_{m s}+R_{e} M_{m s}+\left(R_{e} R_{m s}+L_{e} / C_{m c}+(B l)^{2}+B l K_{p}\right)\left(\gamma \tau_{d}+\beta \tau_{g}\right)+R_{e} / C_{m c}\left(\gamma \beta \tau_{d} \tau_{g}\right) \\
& b_{1}=R_{e} R_{m s}+L_{e} / C_{m c}+(B l)^{2}+B l K_{p}+R_{e} / C_{m c}\left(\gamma \tau_{d}+\beta \tau_{g}\right) \\
& b_{0}=R_{e} / C_{m c}
\end{aligned}
$$

2 The methodology of lead-lag compensator design is therefore to place 3 additional pole and zero so that a positive or negative phase may be properly 4 added at the desired crossover frequencies. By phase crossover frequency, 5 we mean the frequency where phase shift is equal to $-\pi / 2$, as illustrated in 6 Fig. 4. The first step consists in estimating the frequency response function 7 of the uncompensated system, and then determine the amount of postive 8 phase needed to satisfy the phase margin requirements. Likewise, the lag and stability are not much affected. 


\section{1}

2

3

4

5

6

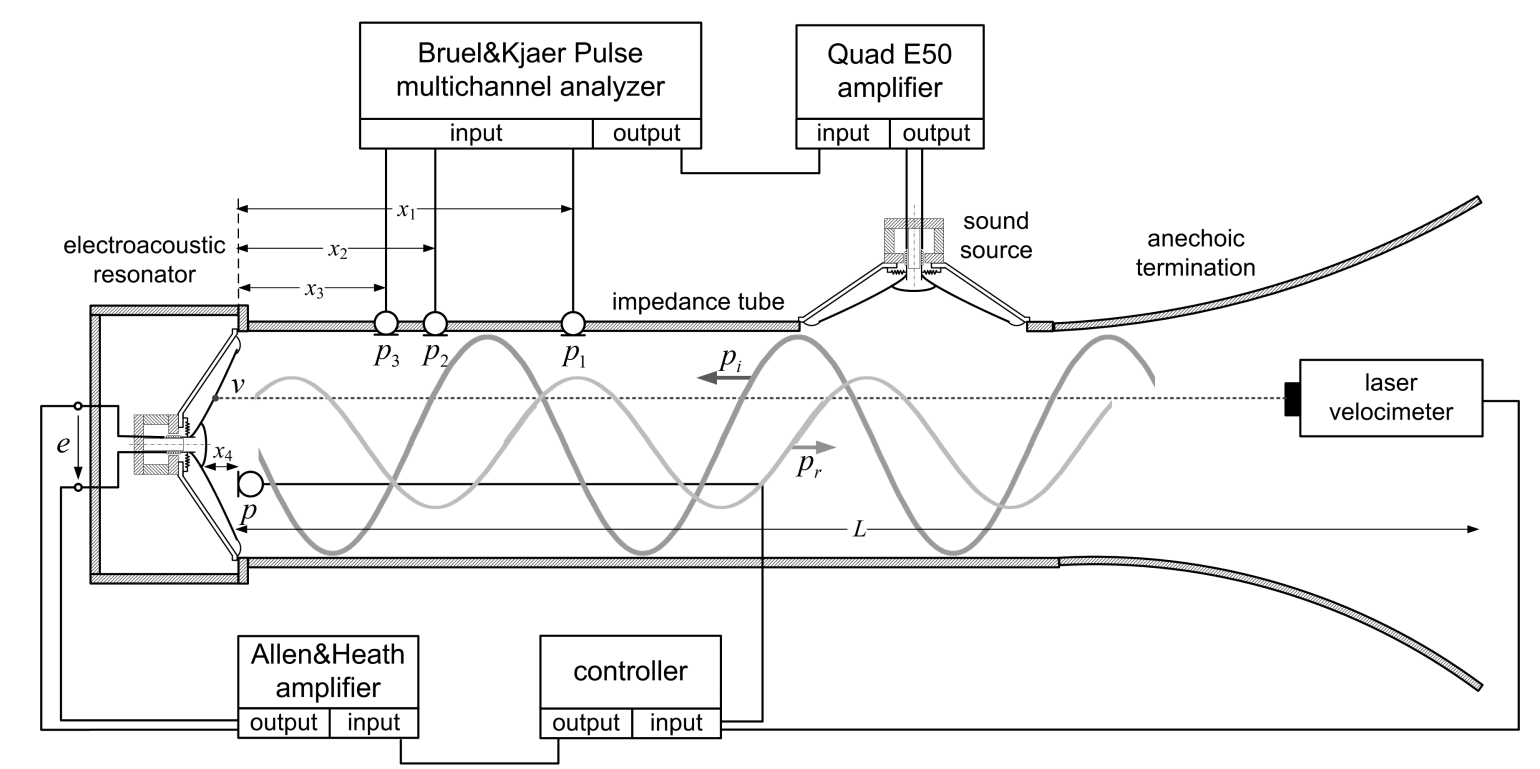

\subsection{Experimental setup}

The experimental setup is shown in Fig. 7. An impedance tube has been specifically designed (length $L=3.4 \mathrm{~m}$ and internal diameter $\varnothing=150$ $\mathrm{mm}$ ), one termination being closed by an electroacoustic resonator (ER). The excitation is generated by a source loudspeaker which is wall-mounted close to the other extremity that is open with a horn shape.

Figure 7: Schematics of the experimental setup.

8

9

10 -

"

n

The specific acoustic admittance ratio $\rho c Y$ and absorption coefficient $\alpha$ are assessed after ISO 10534-2 standard [31]. Three holes located at positions $x_{1}$ $=0.8 \mathrm{~m}, x_{2}=0.46 \mathrm{~m}$, and $x_{3}=0.35 \mathrm{~m}$ from the ER are the receptacles of $1 / 2 "$ microphones (Norsonic Type 1225 cartridges mounted on Norsonic Type 1201 amplifier) sensing the sound pressure $p\left(x_{1}\right), p\left(x_{2}\right)$ and $p\left(x_{3}\right)$. The transfer 
1 functions $H_{13}=p\left(x_{3}\right) / p\left(x_{1}\right)$ and $H_{23}=p\left(x_{3}\right) / p\left(x_{2}\right)$ are processed through a 2 Pulse Brüel and Kjær multichannel analyzer. For the simulations and the 3 design of the feedback control prototypes, a Visaton ${ }^{\circledR}$ AL-170 loudspeaker 4 has been chosen as the electroacoustic resonator prototype, the Thiele-Small 5 parameters of which are given in Tab. 1. We also consider a sealed enclosure 6 loading the rear face of the electroacoustic resonator, the volume of which 7 is $10 \mathrm{dm}^{3}$. In this setup, the front radiation impedance is neglected and the s control law then only accounts for the physical parameters of the loudspeaker 9 model. This simplification will be challenged with experimental results in the following lines.

\subsection{Control system implementation}

The electroacoustic resonator velocity required in the feedback loop is sensed with a Polytec OFV-505/5000 laser velocimeter. As depicted in Fig. 7. the velocity sensor is positioned at the termination of the duct, the laser beam focusing on a single point of the diaphragm, supposedly moving as a rigid piston. The pressure $p$ used in the control loop is sensed with a PCB 130D20 microphone, located at $x_{4}=5 \mathrm{~mm}$ from the diaphragm and slightly off-center at a height of $3.2 \mathrm{~cm}$ from the duct wall. The digital controller used to apply the control parameters and to mix the input signals is implemented on a real-time CompactRIO ${ }^{\circledR}$ (National Instrument) platform supporting FPGA technology. The control signal $e$ is amplified with a unit gain before being applied to the terminals of the electroacoustic resonator. Table 2 summarizes the control parameters used for running the simulation and measurement. 
Table 2: Control settings used for running the simulation and measurement.

\begin{tabular}{|c|c|c|c|c|c|c|c|c|c|}
\hline \multirow{2}{*}{ Topology } & \multirow{2}{*}{ Case } & \multicolumn{3}{|c|}{ PID parameters } & \multicolumn{5}{|c|}{ Phase lead/lag parameters } \\
\hline & & $K_{p}$ & $T_{i}$ & $T_{d}$ & $\alpha$ & $\tau_{d}$ & $K_{c}$ & $\beta$ & $\tau_{g}$ \\
\hline $\mathrm{P}$ control & $\mathrm{A}$ & 20 & & & & & & & \\
\hline \multirow{3}{*}{ PID control } & $\mathrm{B}$ & 20 & 0.025 & & & & & & \\
\hline & $\mathrm{C}$ & 20 & & 0.0001 & & & & & \\
\hline & $\mathrm{D}$ & 20 & 0.025 & 0.0001 & & & & & \\
\hline \multirow{3}{*}{$\begin{array}{l}\text { Lead/lag } \\
\text { compensator }\end{array}$} & $\mathrm{E}$ & 20 & & & 40 & 0.001 & 0.0024 & & \\
\hline & $\mathrm{F}$ & 20 & & & & & 0.24 & 0.01 & 0.002 \\
\hline & $\mathrm{G}$ & 20 & & & 40 & 0.001 & 0.24 & 0.01 & 0.002 \\
\hline
\end{tabular}

\subsection{Acoustic performances assessment}

2 The following results show the measured and computed acoustic per3 formance of an electroacoustic resonator (ER) with different active control 4 strategies, under normal-incidence plane waves. Figures 8 and 9 show the 5 computed and measured frequency response (Bode plot) of the ER in open 6 circuit, i.e. with no control, and for the control settings listed in Tab. 2 . 7 The examination of these results shows that the measured specific acous8 tic impedances are satisfactorily consistent with the corresponding model. 9 Slight differences can be attributed to imperfections in the lumped parameter model that, among other things, neglects the radiation impedance, as well as the frequency response function of sensors not taken into account in the model. In the following, all experimental results obtained with the different control strategies are compared to a baseline configuration consisting of the 
electroacoustic resonator in open circuit.
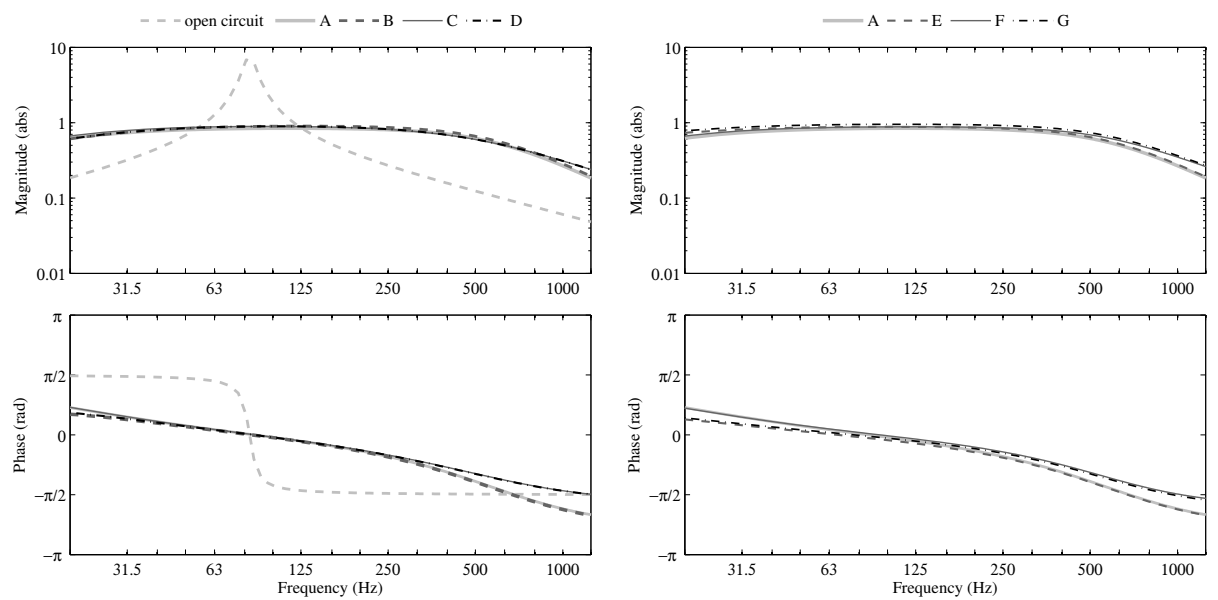

(a)

(b)

Figure 8: Frequency response function of the normalized specific acoustic admittance $y_{P I D}=\rho c Y_{P I D}$ and $y_{\text {lead } / l a g}=\rho c Y_{\text {lead } / \text { lag }}$ computed when the electroacoustic resonator is under PID control (a) and under proportional control including phase compensation (b).

2

When no control voltage is applied (light grey dashed lines), the electroacoustic resonator behaves as a passive, second order bandpass system. The dynamic system is controlled by its stiffness at low frequencies (thus the admittance is proportional to frequency), then presents a maximum at resonance where it is controlled by resistances, and it is controlled by its mass above resonance (thus the admittance is inversely proportional to frequency). The phase shift between the driving pressure and output diaphragm velocity starts from $\pi / 2$ at low frequencies, decreases to zero at the natural resonance of the loudspeaker, and ends up at $-\pi / 2$ at high frequencies. The action of proportional control (case A) consists in adding an equivalent mechanical resistance to the loudspeaker diaphragm so as to match the characteristic 
impedance $\rho c S$ of the medium. Thus, the magnitude of the normalized ad2 mittance equals almost unity over a large frequency range whilst the phase 3 remains close to zero. Unfortunately, the application of a proportional feed4 back gain also increases the overall phase lag which is mainly due to the 5 voice-coil inductive effects. As can be seen in Figure 8 (case A), the measured 6 phase shift between the driving pressure force and the output velocity crosses $7-\pi / 2$ at roughly $550 \mathrm{~Hz}$, which means that the closed loop system is not 8 strictly dissipative above that frequency.

9 The implementation of advanced control systems helps mitigate this 10 overshoot. The phase lead effect generated by the derivative control (case ${ }_{11}$ C) for instance, may compensate the phase shift induced by the voice-coil 12 inductive effect. Adding a lead compensator into the control system (case F)

is another way to compensate the inductive effect, as shown in Fig. 8(b) and 9(b). These two advanced control schemes enable the phase of the acoustic admittance to be kept between $\pm \pi / 2$, thus preventing the ER from becoming over-reflective. This strategy then allows the frequency range over which the ER remains a dissipative system to be extended. However, it may reduce the robustness of the closed loop if some characteristics of the system dynamics are not accurately modeled, particularly as regards higher modes of the diaphragm. Therefore, there is a tradeoff to find between a fast tracking of the reference signal which induces a wider bandwidth, and the sensitivity to underestimated parameters.

The phase lag effect generated by integral control is expected to reduce the phase of the acoustic admittance at low frequencies, as shown in Fig. $5(a)$. This phase compensation can be beneficial in the context of impedance 

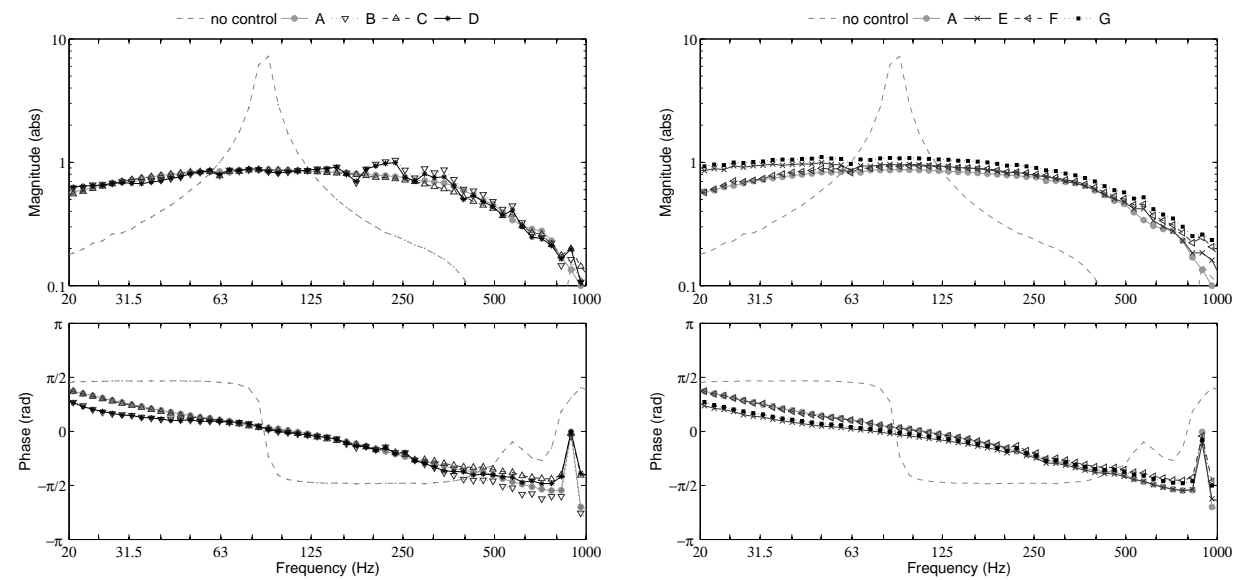

(a)

(b)

Figure 9: Frequency response function of the normalized specific acoustic admittance measured when the electroacoustic resonator is under PID control (a) and under proportional control including phase compensation (b).

1 matching, by extending the bandwidth of the ER below its natural resonance 2 (case B). The absorption coefficient of the diaphragm is then improved at low 3 frequencies, as illustrated in Fig. 10(a). Unfortunately, integral control also 4 increases the phase shift of the system above the loudspeaker resonance, which 5 may negatively affect the performances compared to the proportional control. 6 As a result, the unwanted behavior where the ER is no longer a dissipative 7 system is amplified, as shown in Fig. 8(a) and 9(a). Using a lag compensator 8 (case E) produces a similar affect to applying integral control but without 9 generating excessive phase lag to the ER above its natural resonance. As 10 shown in Fig. 8(b) and 9(b), the phase response in the Bode plot with lag 11 compensation is close to that of proportional control (case A), except for very 12 low frequencies. From this point of view, the combination of proportional 
control and lag compensation should be preferred over integral control. As can be expected, PID control (see case D) can achieve the target acoustic resistance while compensating the phase lag due to the voice-coil inductive effect that affects performance obtained with proportional control. Such an improved absorption can also be achieved by combining proportional control and phase compensation (see case G).

\subsection{Extending the frequency range of absorption}

Figure 10 illustrates the performance in terms of acoustic absorption measured on an ER using PID control (a) and lead/lag compensation (b). It can be clearly seen that applying PID control further extends the bandwidth of the ER. Unlike proportional control (case A), which can be identified with "active damping", integral control (case B) results in the extension of the absorption capability below $60 \mathrm{~Hz}$, the action of the integral control being assimilated to "active stiffness". However, the diaphragm can become more reflective than a rigid wall above $550 \mathrm{~Hz}$. Using derivative control (case C), conversely, the sound absorption coefficient always remains positive above 550 $\mathrm{Hz}$, meaning that the ER is still a dissipative system for the whole frequency span, as shown in Fig. 10 (a). The action of the derivative control can then be assimilated to "active mass". Furthermore, it can be observed that PID control (case D) leads to an extension of the absorption bandwidth while preventing the ER from feeding back more acoustic energy than it receives even at higher frequencies. In this case, the system adds the same percentage of active stiffness (integral action) and active mass (derivative action), and hence the corresponding phase lag and phase lead effects are balanced. In summary, PID control, i.e. an active "damping-stiffness-mass" system that 
improves the absorption performances of the ER below, at, and above the 2 loudspeaker resonance without inducing adverse effects in the other regions. 3 Lastly, the use of a phase lead compensation (case C) can keep the phase of 4 the acoustic admittance frequency response function bounded between $\pm \pi / 2$. 5 It is therefore beneficial in terms of sound absorption to combine a phase lead 6 compensator with a phase lag compensator, as shown in case G.

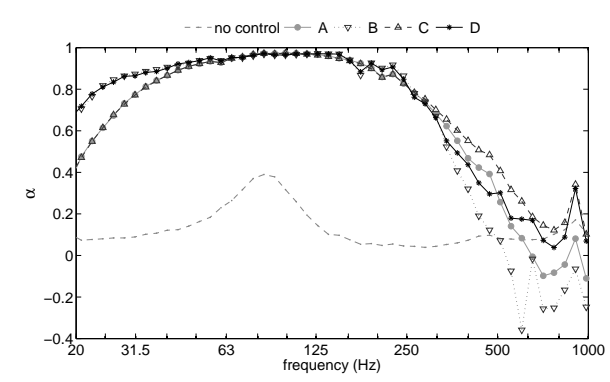

(a)

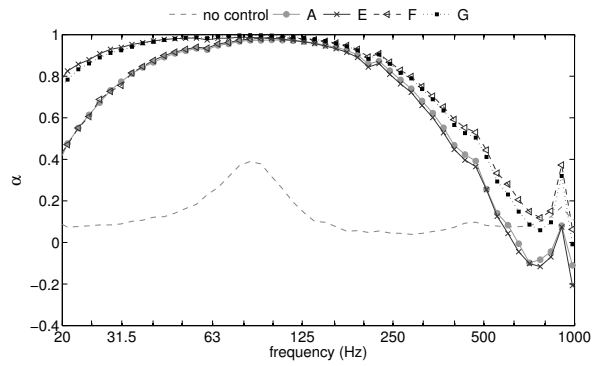

(b)

Figure 10: Acoustic absorption coefficient measured when the electroacoustic resonator is under PID control (a) and under proportional control including phase compensation (b). \\ 5.5. Overall stability when coupling with a resonant sound field}

The absolute stability of the active electroacoustic resonator has been discussed so far. In practice, however, stability issues can still be experienced due to the coupling of the active device with the potentially resonant sound field. This is mainly due to the presence of reactive components in the loudspeaker system such as the coil inductance or alternatively the higherorder resonances of the diaphragm which are not taken into account in the model. When placed in an enclosure such as a duct, or even in a room, the ER can interact with the resonant sound field and cause positive acoustic 
1 feedback [32]. This may arise when increasing too much the feedback gain, 2 thus limiting the absorbing performances of the ER. The measured open-loop 3 frequency response of the system, from the loudspeaker's input voltage to 4 controller output voltage, is used here to quantify the relative margin of 5 stability of the ER. The Nyquist plot and Bode plot of the open-loop gain are 6 both shown in Fig. 11. In a first step, the crossover frequencies, which are 7 the frequencies at which the argument of the open-loop gain equals $-\pi$, are 8 identified. Then, phase and gain margins [28] are evaluated, determining the 9 parameter changes margins that can be accepted before the feedback system 10 becomes marginally stable. Since there are multiple crossover frequencies in

the case of the studied dynamical system, the one with the smallest phase margin dictates the overall stability margin. This value has been measured for various control settings and listed in Tab. 3 .

Table 3: Summary of the measured stability margins when the ER is radiating in the duct.

\begin{tabular}{lccc} 
Topology & Case & Gain margin & Phase margin \\
\hline P control & A & $8.0 \mathrm{~dB}$ at $2430 \mathrm{~Hz}$ & $55.6^{\circ}$ at $686 \mathrm{~Hz}$ \\
PID control & D & $11.6 \mathrm{~dB}$ at $2708 \mathrm{~Hz}$ & $65.3^{\circ}$ at $686 \mathrm{~Hz}$ \\
Lead/lag compensator & G & $8.7 \mathrm{~dB}$ at $2692 \mathrm{~Hz}$ & $46.6^{\circ}$ at $689 \mathrm{~Hz}$ \\
\hline
\end{tabular}

14 As shown in Tab. 3, the stability margin is comfortable for each of the assessed control settings. It can be noted that the use of a PID control scheme (case D) induces slightly higher stability margin compared to the others settings. Increasing the feedback gain $K_{p}$, obviously, reduces the stability margin of the coupled system. As already mentioned in [4] the resonances of the duct represent the most important factor of magnitude and 
phase variations in the open-loop gain. It is especially worth noting that the 2 stability margins are significantly increased when the ER is placed in free 3 field conditions.

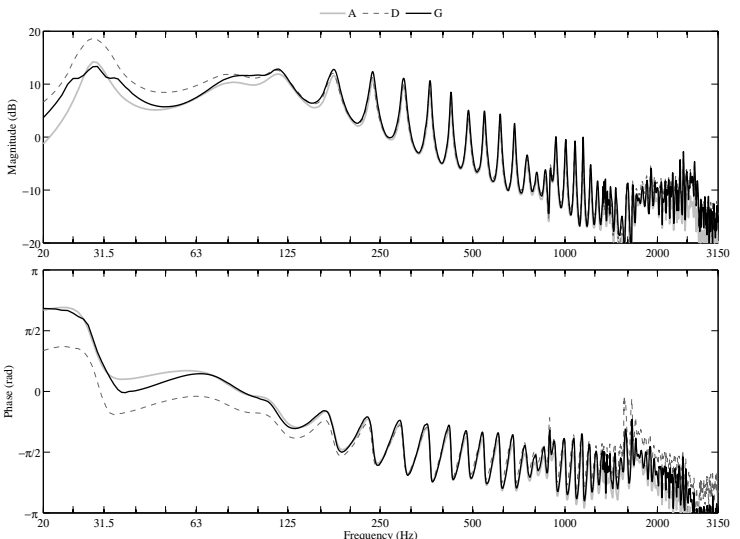

(a)

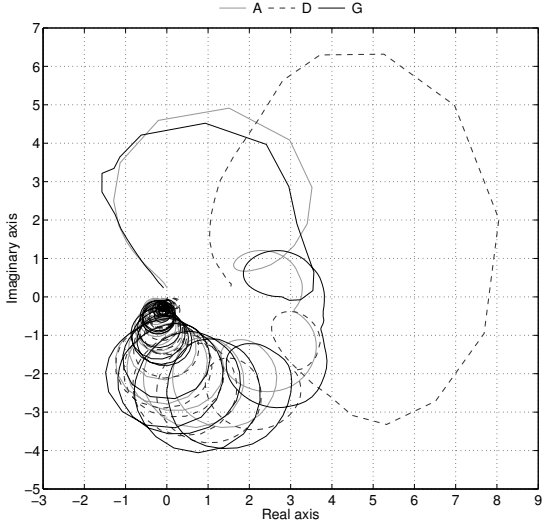

(b)

Figure 11: Open-loop frequency response of the ER when coupled to a resonant sound field (duct) for various control settings. The Bode plot and Nyquist diagram of the transfer function measured from the loudspeaker input voltage to controller output voltage are shown on the left- and right-hand side, respectively.

4 5.6. Active damping of modal resonances in a duct

5 To assess the control performance for damping low-frequency modal reso6 nances, the ER is installed at one end of a $2.6 \mathrm{~m}$ length duct, the other end 7 being let open. A sound source wall-mounted near the open end delivers a 8 swept sine excitation. A microphone located at position $x_{2}=0.46 \mathrm{~m}$ from the 9 ER is used to pick up the measured sound pressure inside the duct. Figure 12 illustrates the magnitude response of the measured sound pressure to applied 11 voltage, expressed in $\mathrm{dB}$ re. $20 \mu \mathrm{Pa} \mathrm{V}^{-1}$, when the duct is ended by a hard wall and by an ER with various control settings. 


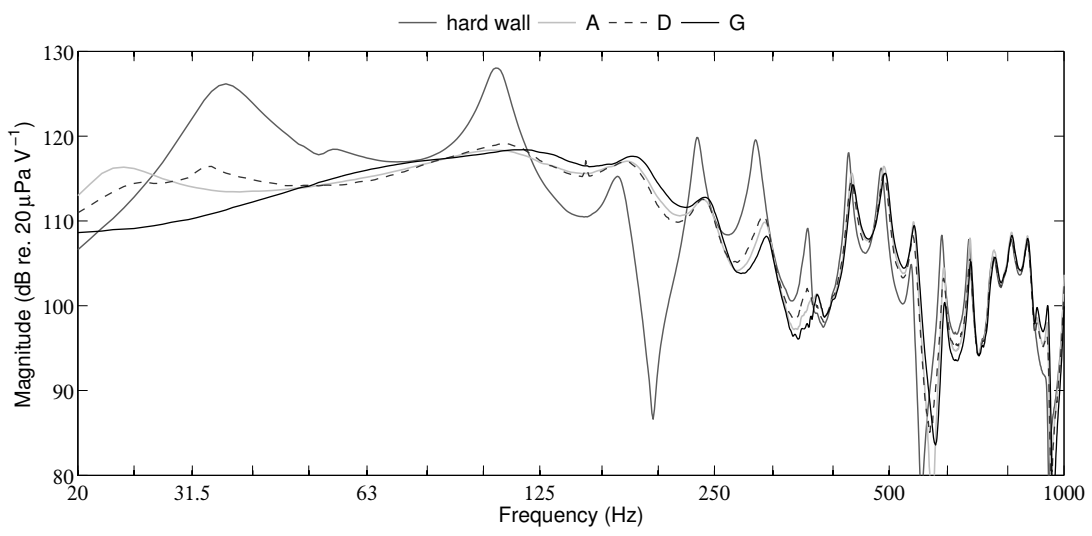

Figure 12: Sound pressure level measured in the duct with respect to the voltage applied to the sound source ; case A (proportional control), case D (PID control), and case G (proportional control including phase compensation)

$1 \quad$ As expected, a sound field with low modal density and strong resonances 2 can be observed in the duct when it is ended with a hard wall. As shown in 3 Fig. 12, the low-frequency modal resonances of the duct can be significantly 4 attenuated by the different control strategies. The acoustic gain is computed 5 as the difference between the sound pressure level measured at position $x_{2}$ with 6 the baseline hard wall termination and the one measured with the different 7 active ER settings. The acoustic gain achieved using proportional control 8 (case A) is $-13 \mathrm{~dB}$ for the first mode at $36 \mathrm{~Hz},-9.7 \mathrm{~dB}$ for the second mode at 9 $105 \mathrm{~Hz}$, and between $-3 \mathrm{~dB}$ and $-10 \mathrm{~dB}$ with the following ones, up to 500

Hz. Applying PID control (case D) or adding a lead/lag compensator (case G) can even further improve the acoustic gain. With case G for instance, the measured gain is $-15 \mathrm{~dB}$ for the first mode of the duct. The damping of the low-frequency resonances is also slightly enhanced for the peaks around $620 \mathrm{~Hz}$ and $690 \mathrm{~Hz}$ for case $\mathrm{G}$, of order of 3-4 dB compared with a purely 
1 proportional control (case A). The magnitudes of acoustic resonances and 2 anti-resonances can therefore be minimized through active electroacoustic 3 resonators, thereby equalizing the distribution of sound energy in enclosed 4 sound fields. This effect is particularly desired in the listening space such as 5 the control room of recording studios, home theaters or even concert halls, 6 to provide the best sound reproduction quality. Nevertheless, in the case of $73 \mathrm{D}$ modes, the target acoustic impedance may differ from the characteristic 8 impedance of the air (which only corresponds to the matching condition for 9 normal incidence plane waves), and the reported strategy may not provide the 10 optimal mode reduction. However, preliminary experiments show that such an active electroacoustic resonator strategy also provides significant mode damping in $3 \mathrm{D}$ contexts, even though the condition on impedance matching is not always well optimized [8].

\section{Conclusions}

This paper discussed a simple engineering approach to achieve active sound absorption using feedback-controlled loudspeakers. The control objective consists in adjusting the acoustic resistance of the membrane absorber to a target value, and then expand the bandwidth over which the acoustic impedance is properly matched to the characteristic impedance of the medium. The benefit of using PID control or combining phase compensation and proportional control is also discussed. It is shown that some adverse effects, due to high-order features in the dynamics of the moving-coil loudspeaker, can be mitigated with corrective actions. Improved acoustic performance are achieved in terms of bandwidth, in addition to ensuring that the ER strictly remains a dissipative 
system over the whole frequency range of interest. This work gives an insight into the ability of electroacoustic resonators to achieve sound absorption in enclosed sound fields, specifically in low frequencies for which conventional passive materials have poor absorbing capabilities. It provides comprehensive information on the design of basic control schemes, drawing on classical control techniques, that allows conventional electrodynamic loudspeakers to be turned into sound absorbers. The proposed model has been developed under the small movement hypothesis. Thus, improved models accounting for nonlinear behaviour, that might arise with more demanding conditions, should be considered in future work. Further studies will also address the implementation of such active electroacoustic resonators for the damping of room modes in actual listening rooms, with a view to validating these strategies for real-life applications, such as the low-frequency equalization of recording studios.

\section{Acknowledgments}

This research work was supported by the Swiss National Science Foundation (SNSF), under grant agreement 200020-132869.

The authors would like to thank Patrick Roe for his help in proofreading the paper.

\section{Appendix A.}

This appendix summarizes the method, described in [26, 27], for solving a cubic equation. This technique is specifically employed to derive the poles in the expression of the acoustic admittance of the electroacoustic resonator. 
1 Let us consider a cubic polynomial of the form

$$
D(s)=b_{3} s^{3}+b_{2} s^{2}+b_{1} s+b_{0}
$$

2 A set of compact algebraic formulae based on hyperbolic functions can 3 be derived for evaluating the roots of the cubic equation. Let $N\left(s_{N}, t_{N}\right)$ be ${ }_{4}$ the point of inflection on the curve plotted in Fig. A.13, i.e. the point on the 5 polynomial curve $D(s)$ such that shifting the $x$-axis by putting $z=s-s_{N}$ 6 makes the sum of the roots of the new polynomial $D(z)$ equal zero. As can be 7 seen in Fig. A.13, $N$ is the point of symmetry of the cubic function such that $8 s_{N}=-b_{2} /\left(3 b_{3}\right)$ and $t_{N}=f\left(s_{N}\right)$. The sideways $S$-shape is typical of what 9 should be expected for the shape of a cubic function. The cubic function has two turning points; it rises toward positive infinity in one direction and drops toward negative infinity in the other end. Let the parameters $\delta, \lambda$, and $h$ be

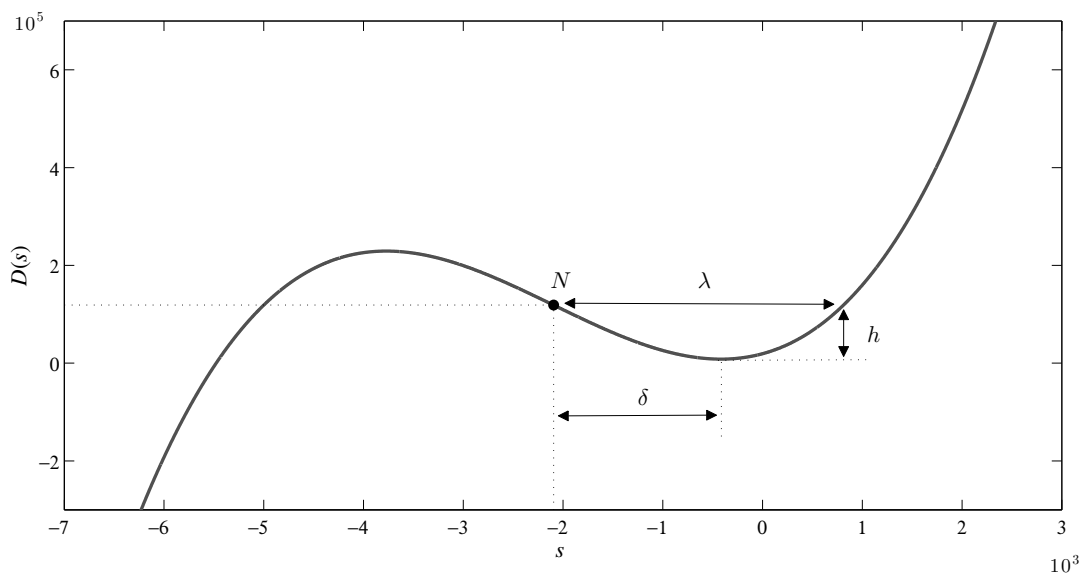

Figure A.13: Characteristic curve describing the numerator of the loudspeaker transfer function as a specific cubic polynomial. 
1 defined as the distances in Fig. A.13, By locating the turning points, it can 2 be shown that $\lambda$ and $h$ are simple functions of $\delta$, as

$$
\delta^{2}=\frac{b_{2}{ }^{2}-3 b_{3} b_{1}}{9 b_{3}{ }^{2}} \quad \lambda^{2}=3 \delta^{2} \quad h=2 b_{3} \delta^{3}
$$

3 Thus, the shape of the cubic function is completely characterized by 4 the parameter $\delta$. Three cases have to be considered. If $\delta^{2}>0$ the cubic 5 exhibits distinct maxima and minima, if $\delta^{2}=0$ both points are coincident, 6 and if $\delta^{2}<0$ there are no turning points. As illustrated in Fig. A.13, the 7 characteristic equation of a conventional loudspeaker is such that $\delta^{2}>0$. The 8 curve crosses the $x$-axis only once, meaning that this particular cubic exhibits 9 one real root. In addition, it reveals that $t_{N} / h>1$. In that case, the roots of the original cubic polynomial can be derived using a set of compact algebraic formulae based on hyperbolic functions, as

$$
\begin{aligned}
& p_{1}=s_{N}-2 \delta \cosh \left(1 / 3 \cosh ^{-1}\left(t_{N} / h\right)\right) \\
& p_{2}=s_{N}+\delta\left[\cosh \left(1 / 3 \cosh ^{-1}\left(t_{N} / h\right)\right)+i \sqrt{3} \sinh \left(1 / 3 \cosh ^{-1}\left(t_{N} / h\right)\right)\right] \\
& p_{3}=s_{N}+\delta\left[\cosh \left(1 / 3 \cosh ^{-1}\left(t_{N} / h\right)\right)-i \sqrt{3} \sinh \left(1 / 3 \cosh ^{-1}\left(t_{N} / h\right)\right)\right]
\end{aligned}
$$

12 Finally, the cubic polynomial ( A.1) can be rewritten more compactly as

$$
D(s)=\left(s-p_{1}\right)\left(s-p_{2}\right)\left(s-p_{3}\right)
$$

13

14

15

\section{References}

[1] T. J. Cox, P. D'Antonio, Acoustic absorbers and diffusers, Taylor and Francis, 2nd ed., pp. 419-439, (2009). 
1 [2] R. Mariani, S. Bellizzi, B. Cochelin, P. Herzog and P.O. Mattei, Toward $2 \quad$ an adjustable nonlinear low frequency acoustic absorber, J. Sound and $3 \quad$ Vib., 330(22), pp. 5245 - 5258, 2011

4 [3] A.J. Fleming, D. Niederberger, S.O.R. Moheimani and M. Morari, Control 5 of resonant acoustic sound fields by electrical shunting of a loudspeaker, $6 \quad$ IEEE Transaction on Control Systems Technology, 15(4), 2007.

7 [4] H. Lissek, R. Boulandet, R. Fleury, Electroacoustic absorbers: bridging the $8 \quad$ gap between shunt loudspeakers and active sound absorption, J. Acoust. $9 \quad$ Soc. Am., 129(5), pp 2968-2978, 2011.

[5] R. Boulandet and H. Lissek, Optimization of electroacoustic absorbers by means of designed experiments, Applied Acoustics, 71(9), pp. 830-842, 2010.

[6] T. Sluka, P. Mokrý and H. Lissek, A theory of sound transmission through a clamped curved piezoelectric membrane connected to a negative capacitor, Int. J. of Solids and Structures, 47(17), pp. 2260 - 2267, 2010.

[7] H. Lissek and X. Meynial, A preliminary study of an isodynamic transducer for use in active acoustic materials, Applied Acoustics, 64(9), pp. 917-930, 2003.

[8] H. Lissek, R. Boulandet, E. Rivet and I. Rigas. Assessment of active electroacoustic absorbers as low-frequency modal dampers in rooms, Internoise 2012, New-York City, USA, August 19-22, 2012.

[9] H. Kuttruff, Room acoustics, Spon Press, 5th ed., 2009. 
1 [10] S. J. Elliott, A. R. D. Curtis, A. J. Bullmore, P. A. Nelson, The active 2 minimization of harmonic enclosed sound fields, part 3: Experimental $3 \quad$ verification, J. Sound and Vib., 117(1), 1987.

4 [11] S. J. Elliott, P. Joseph, P. A. Nelson and M. E. Johnson, Power output 5 minimization and power absorption in the active control of sound, J. $6 \quad$ Acoust. Soc. Am., 90(5), pp. 2501-2512, 1991.

7 [12] P. A. Nelson, A. R. D. Curtis, S. J. Elliott, A. J. Bullmore, The active $8 \quad$ minimization of harmonic enclosed sound fields, part 1: Theory, J. Sound $9 \quad$ and Vib., 117(1), 1987.

[13] P. A. Nelson, S. J. Elliott, Active Control of Sound, Academic Press Inc, 1993.

[14] M. Furstoss, D. Thenail, M.A. Galland, Surface impedance control for sound absorption: direct and hybrid passive/active strategies, J. Sound and Vib., 203(2), pp. 219-236, 1997.

[15] M. Melon, P. Herzog, A. Sitel and M.-A. Galland, One dimensional study of a module for active/passive control of both absorption and transmission, Applied Acoustics, 73(3), pp. 234-242, 2012.

[16] H. W. Bode, Network analysis and feedback amplifier design, Van Nostrand, Princeton, 1945.

[17] J. Vanderkooy, A model of loudspeaker driver impedance incorporating Eddy current in the pole structure, J. Audio Eng. Soc., 37(3), 1989. 
1 [18] W. Klippel, Nonlinear large-signal behavior of electrodynamic loudspeak2 ers at low frequencies, J. Audio Eng. Soc., 40(6), 1992.

3 [19] H. H. Woodson, J. R. Melcher, Electromechanical dynamics, John Wiley $4 \quad \&$ Sons, 1968.

${ }_{5}[20]$ R. H. Small, Direct radiator loudspeaker system analysis, J. Audio Eng. $6 \quad$ Soc., 20)(5), pp. 383-395, 1972.

7 [21] M. Rossi, Audio, Presses Polytechniques et Universitaires Romandes, $8 \quad 2007$.

9 [22] P. M. Morse, K. U. Ingard, Theoretical acoustics, Princeton University 10 Press, 1968

11 [23] L.L. Beranek, Acoustics, McGraw-Hill, 1954, p. 11.

12 [24] W. Frommhold, H. V. Fuchs, S. Sheng, Acoustic performance of mem${ }_{13} \quad$ brane absorber, J. Sound and Vib., 170(5), pp. 621-636, 1994.

14 [25] M. J. Turner, D. A. Wilson, The use of negative source impedance with 15 moving coil loudspeaker drive units: an analysis and review, J. Audio Eng. $16 \quad$ Soc., 122th Convention, paper 7072, Vienna, Austria, 2007.

17 [26] R. W. Nickalls, A new approach to solving the cubic: Cardan's solution revealed, The Mathematical Gazette, 77(480), 1993.

[27] G. C. Holmes, The use of hyperbolic cosines in solving cubic polynomials, ${ }_{20} \quad$ The Mathematical Gazette, 86(507), 2002.

21 [28] K. Ogata, Modern control engineering, Prentice-Hall, Inc, 3rd Ed., 1997. 
1 [29] E. Brogliato, R. Lozano, B. Maschke, O. Egeland, Dissipative system analysis and control: Theory and applications, Springer, 2007.

3 [30] A. Visioli, Practical PID control, Springer, 2006.

4 [31] ISO 10534-2, Acoustics - Determination of sound absorption coefficient 5 and impedance in impedance tubes - Part 2: Transfer-function method, $6 \quad$ ISO, Geneva, Switzerland, 1998.

7 [32] R. L. Clark, K. D. Frampton, D. G. Cole, Phase compensation for feedback $8 \quad$ control of enclosed sound field, J. Sound Vib., 195(5), pp. 701-718, 1996. 